\title{
HOLISTIC MONITORING OF MACHINING SYSTEM
}

\author{
Gabriel Frumuşanu, Alexandru Epureanu \\ “Dunărea de Jos” University of Galaţi, Department of Manufacturing Engineering \\ Domnească Street 111, 800201 - Galaţi, Romania
}

Corresponding author: Gabriel Frumuşanu, gabriel.frumusanu@ugal.ro

\begin{abstract}
Nowadays, the part program describes only the process itself and not the obtained performance. The operator monitors just some of the variables describing the actually obtained product and appropriately adjusts the values of the programmed variables. This adjustment is realised with a considerable delay and without an adequate fundament (many times even intuitively). Moreover, process monitoring currently follows only to notice the occurrence of perturbations and, hence, of deviations from process plan. As consequence, the performance in accomplishing the manufacturing task might be diminished due to an insufficient knowledge about both the system dynamics and the conditions in which the process is performed. Starting from these premises, the challenge addressed here is to rebuild at conceptual level the monitoring system such us the monitoring becomes holistic, this meaning evaluation \& reveal of machining system current state \& dynamics. In other words, the holistic monitoring concerns both the values of the variables describing the system state and the relations of causality between them. In this paper, the holistic monitoring is introduced through an illustrative sample. The monitoring variables and functions are defined and sampled.
\end{abstract}

Key words: holistic monitoring, machining operation, machining system dynamics, online learning, holonic modeling.

\section{LIST OF NOTATIONS}

$T_{n} \quad$ received/released task

$T_{\alpha} \quad$ assumed task

$D_{\alpha} \quad$ deliverable

$T(i) \quad$ task of $i$ class

$\left\{t_{k}(i)\right\} \quad$ set of variables describing $T(i)$

$T L(i, j) \quad$ tooling of $j$-version able to support the accomplishment of $T(i)$ task

$\left\{t l_{k}(i, j)\right\} \quad$ set of variables describing the $T L(i, j)$ tooling

$K L(i, j) \quad$ knowledge supporting the accomplishment of $T(i)$ task by using $T L(i, j)$ tooling, where

$$
K L(i, j)=\left\{\begin{array}{l}
S(i, j) \\
D(i, j) \\
M(i, j)
\end{array}\right\}
$$

$S T(i, j) \quad$ statement including all information regarding $T_{\alpha}$ accomplishment

$D S(i, j) \quad$ dataset resulting by processing a set of $S T(i, j)$ statements

$S(i, j) \quad$ system state when accomplishing $T(i)$ task by using $T L(i, j)$ tooling, with

$$
S(i, j)=\left\{s_{k}(i, j)\right\}
$$

$\left\{s_{k}(i, j)\right\} \quad$ set of state variables describing $S(i, j)$

$D(i, j) \quad$ system dynamics when accomplishing $T(i)$ task by using $T L(i, j)$ tooling, with

$$
D(i, j)=\left\{S_{m}(i, j)\right\}
$$

$\left\{S_{m}(i, j)\right\} \quad$ set of $S(i, j)$ system states ascertained by monitoring

$M(i, j) \quad$ model of the system accomplishing $T(i)$ task by using $T L(i, j)$ tooling, with

$$
M(i, j)=\left\{\begin{array}{c}
\bar{X}(i, j) \\
S D(i, j) \\
a(i, j)
\end{array}\right\},
$$

$\bar{X}(i, j) \quad$ average values of state variables during the accomplishment of $T(i)$ task by using $\operatorname{TL}(i, j)$ tooling

$S D(i, j) \quad$ standard deviation of state variables values during the accomplishment of $T(i)$ task by using $T L(i, j)$ tooling $a(i, j) \quad$ causal model of the system when accomplishing $T(i)$ task by using $T L(i, j)$ tooling

$A L(i, j) \quad$ algorithm enabling the extraction of new $K L(i, j)$ knowledge 


\section{INTRODUCTION}

In the most general sense, monitoring is the action of looking for the evolution of something (machinery, equipment, phenomenon, process etc.), by direct observation or using dedicated measuring devices. Monitoring purpose is to ensure the reaching of a given objective and it may require to take appropriate corrective measures when deviations from the expected evolution are acknowledged.

In manufacturing domain, monitoring may concern the the workpiece features (during its metamorphosis from blank to finished product), the manufacturing equipment condition \& functioning or the manufacturing process performance.

- The workpiece features can be online monitored by on-machine measuring. There are also recent solutions suggesting the use of video cameras in order to monitor the machining errors/ precision (Gostev and Golosov, 2020, Xing et al., 2021) or part deformation and surfaces roughness (Boca and Horodincă, 2020). Unlike the solutions from above, which directly evaluate the machined part, there are also proposed monitoring techniques based on measuring a signal that is subsequently processed in order to extract information concerning the finished product quality. Thus, by measuring the acoustic emission and statistically processing the result, the errors occurred in precision machining processes are monitored (Lee et al., 2006), or the dimensional deviation and specific faults in cold forming processes can also be monitored (Behrens et al., 2017). Multiple solutions for monitoring the results of abrasive finishing processes by measuring acoustic emission, force, vibration and optical signals and processing them by Artificial Neural Networks, multiple regression and fuzzy techniques are available (Pandiyan et al., 2020). Another research examined four signal feature extraction methods to optimize surface finish monitoring by analysis of vibration signals in order to correlate signal statistical measurements with surface roughness (Garcia Plaza et al., 2019).

- Regarding manufacturing equipment condition \& functioning issue, numerous researches addressed tool condition monitoring. More techniques for doing this by recording the cutting force, local temperature or vibration signals, followed by processing the signal through time, frequency or time-frequency domain analysis are presented (Lauro et al., 2014). The same objective is proposed to be reached by measuring the vibration and processing the digitalized signals using reconfigurable band-pass digital filter and statistical techniques (SevillaCamacho et al., 2015), or by identifying the vibration signature in the recorded acoustic semnal (Bhuiyan et al., 2014). Solutions for machine condition monitoring are also present in literature. Thus, datadriven machine health monitoring by deep learning application is suggested (Zhao et al., 2019). A machine tool accuracy condition monitoring scheme using volumetric errors, vector similarity measures and exponentially weighted moving average control chart is proposed (Xing et al., 2019). Manufacturing station health monitoring by vibration signal recording, followed by Virtual Engineering and Discrete Event Simulation can be also mentioned (Assad et al., 2021).

- Despite being of major importance, indicators regarding the manufacturing process performance (consumptions and costs) are rarely addressed as monitoring object. The energy consumption monitored on the base of data-driven models in grinding process is mentioned ( $\mathrm{Lv}$ et al., 2020). Another paper presents a study of using timefrequency representation and deep neural networks to enable real-time, intelligent monitoring of manufacturing process productivity (Liao et al., 2021).

After thoroughly analysing both existing and proposed solutions for machining system monitoring, some shortcomings can be revealed, as belowmentioned.

- Nowadays machining systems are open-loop numerically controlled, according to the part program. The programmed variables describe only the process itself and not the result or the obtained performance.

- The operator monitors just some of the variables describing the obtained product and makes appropriate adjustment of the programmed variables, if needed.

- This adjustment is realised with a considerable delay and without an adequate fundament (many times even intuitively), without knowing the way the system works and its model.

- On the other hand, the currently monitored variables are irrelevant regarding the process performance, which is, in fact, the ultimate goal of any manufacturer.

- The part-program does not keep in view the consumes, which are actually ignored.

- In most of the cases, the information generated by manufacturing process performing is thrown out.

- The dynamics of machining system in time usually is not known.

Starting from here, the challenge to be faced by next generation of machining equipment can be synthesized in three main requirements.

- The part-program should describe the expected results of the machining task instead of the machining procedure parameters ("What" instead of "How"). 
- The information about the machining process ongoing in terms of workpiece attributes and process performance should be online monitored and delivered to machine operator.

- The entire information resulted after each task accomplishment should be recorded, processed and delivered as knowledge, together with the physical result of the task - the product.

The answer to the challenge, requires before anything else to reconsider the machine control issue. The control should be learning-based. For this reason, the monitoring approach is the first thing to be changed. The here-proposed holistic monitoring is the solution. From these premises, this paper target is to rebuild at conceptual level the monitoring system, in order to fructify the opportunities brought by the existence of the global information network. Rebuilding main purpose is to mitigate the risks of efficiency loss in machining system exploitation. The holistic monitoring means evaluation and revealing of machining system current state \& dynamics. In other words, the holistic monitoring concerns both the values of the variables describing the system state and the relations of causality between them. In this paper, the architecture of the holistic monitoring system is detailed through an illustrative example.
The monitoring variables and functions are defined and explained.

In what concerns paper structure, next section presents the manufacturing procedure, involving the manufacturing function and its support. The third section introduces the architecture and the holarchies specific to the manufacturing system of new generation. The fourth section defines and explains the proposed concept of holistic monitoring and also includes an illustrative example, while the last section is for conclusion.

\section{MANUFACTURING PROCEDURE}

\subsection{Manufacturing function}

The manufacturing system function $F$ is to accomplish the manufacturing task $T_{n}$. This means to bring the product from the initial state to the final state, through a manufacturing process, by following a given manufacturing procedure. In mathematical analysis, the functions have argument, analytical expression and value. By making an analogy, in manufacturing function case we can speak about similar notions, namely argument, algorithm and result (Figure 1).

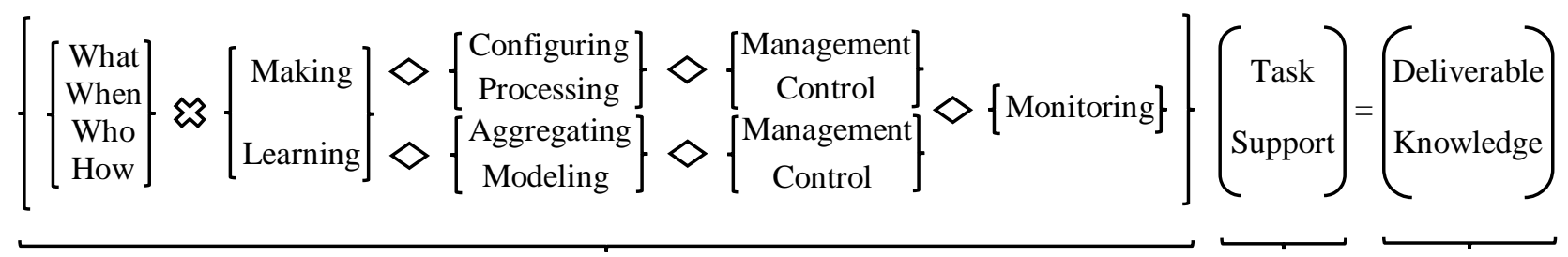

Algorithm (F)

Argument (F) Result (F)

Fig. 1. Manufacturing function

The manufacturing function is applied to a given task, which is accomplished by using a dedicated support, so the couple (task, support) can be seen as argument.

The function result consists in the deliverable specified through the task, but also in the knowledge generated during task accomplishment.

The function algorithm supposes four things to be established [9], namely: what, which refers to task definition, when - to precedence conditions during the manufacturing process, who - to support needed for task accomplishment (humans included), and how - to applied procedure selection.

The algorithm application involves a couple of activities, taking place concomitantly: making and learning. Making refers to actually transforming the product, and consists in configuring and processing. Configuring action concerns both the task, and the support needed for accomplishing it by processing. Task configuring means the repeated division / subdivision of initial manufacturing task among successive generations of manufacturing holons. Support configuring means to assemble all the manufacturing assets following to be used by each holon for its task accomplishment. Learning refers to ascertaining the transformation results. The learned results about product transforming are the subject of aggregating action, which further enables the manufacturing system modeling. The system \& process management is related to configuring and aggregating actions, while their control is related to processing and modeling actions.

For application of manufacturing function algorithm, as above-described, our approach requires holonic modeling of the manufacturing system and holistic monitoring of all involved activities.

\subsection{Function support}

The manufacturing function is accomplished with the support of two types of holons: making-holons 
and learning-holons. Before explaining their functioning procedures, two additional remarks should be made.

- The systematic application of the knowledge concerning the manufacturing processes requires the classification of manufacturing tasks from scheduled tasks pool. Thus, the generic class $i$ includes all the tasks that can be described through the same variables.

- The architectural support pool includes more versions $j$ of both tooling and corresponding supportknowledge that could be used for accomplishing a given task.

The making holon (Figure 2(a)) receives the task $T_{n}$ and, at first, it executes the configuring stage. During this, it assumes from $T_{n}$ the partial task $T_{\alpha}$, which it will actually accomplish, the remaining task being released to other holons from offspring generations. At the same time, it finds $T_{\alpha}$ class $i$ and it configures the support following to be used for $T_{\alpha}(i)$ accomplishment. The support consists in both tooling

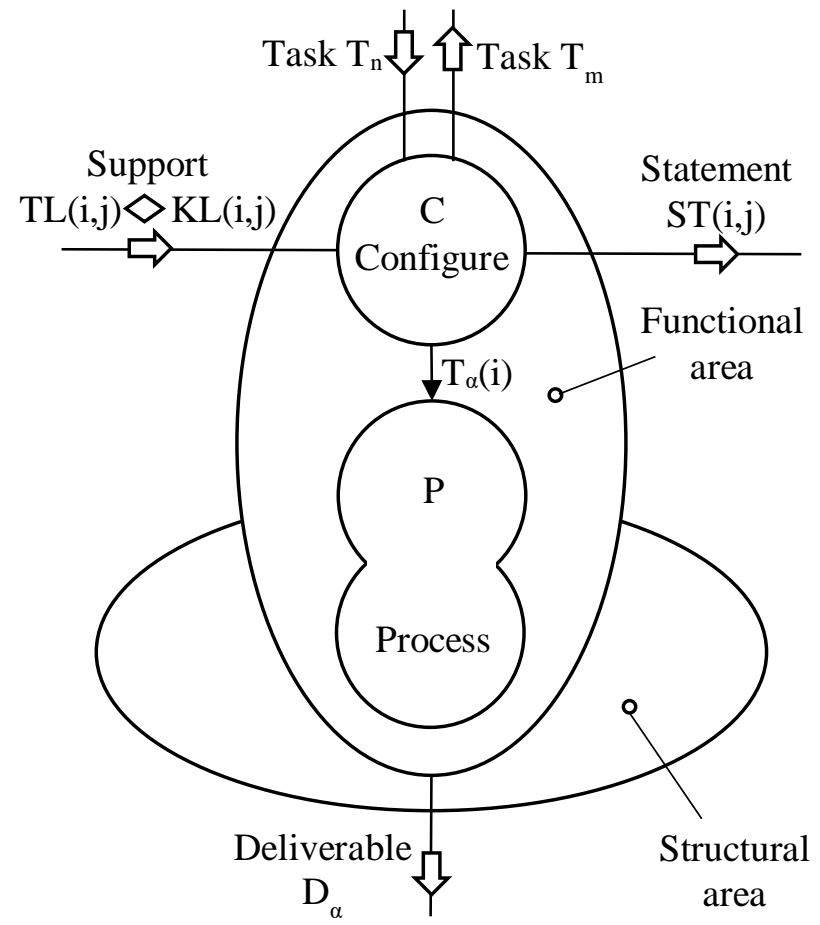

a)
$T L(i, j)$ and knowledge $K L(i, j)$. Then the holon executes the processing stage, during which it accomplishes $T_{\alpha}$, the result consisting in the deliverable $D_{\alpha}$ and the statement $S T(i, j)$ that includes all information regarding $T_{\alpha}$ accomplishment.

The learning-holon (Figure 2(b)) must also execute two stages, namely aggregating and modelling. As input there are a set of $S T(i, j)$ statements coming from the making-holons involved in $T_{n}$ task accomplishment. During aggregating stage, the learning-holon processes the set of statements and generates the dataset $D S(i, j)$. It also selects the needed support, consisting in extraction algorithm $A L(i, j)$ and existing knowledge $K L(i, j)$, which it will apply in modelling stage in order to build the model of $T_{n}$ task accomplishment.

New knowledge $K L(i, j)$ is actually the output delivered by learning-holon as such models.

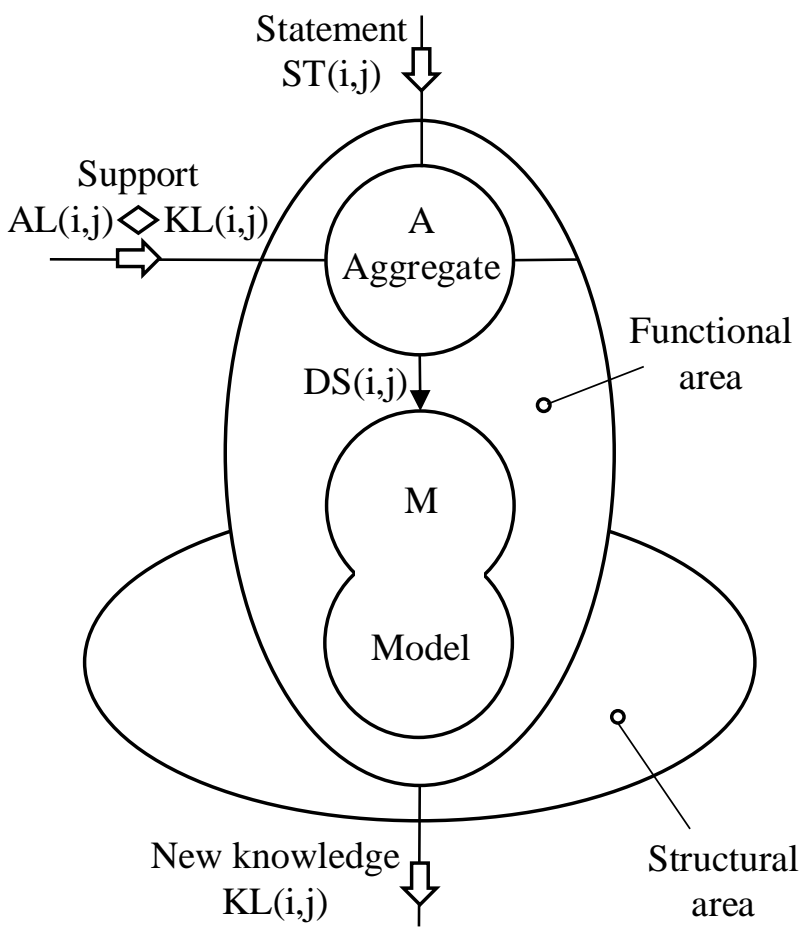

b)

Fig. 2. Manufacturing support:

$\mathrm{a}$ - making-holon, $\mathrm{b}$ - learning-holon

\section{MANUFACTURING SYSTEM}

\subsection{System architecture}

The manufacturing system architecture will be further described. For a better understanding, the simplest case, namely when the system is composed by a unique making-holon and a learning-holon (Figure 3) will be addressed. 


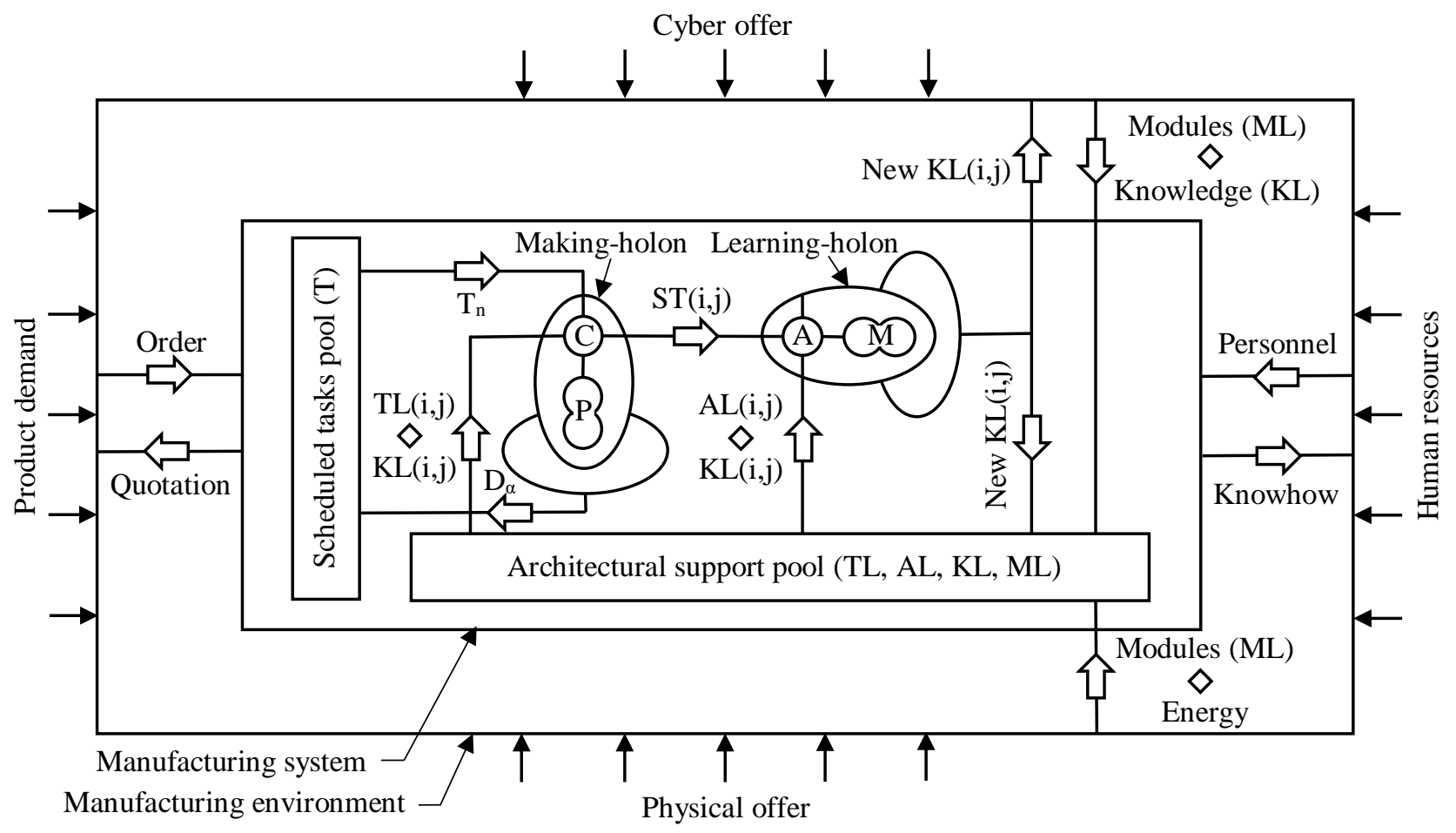

Fig. 3. Manufacturing system architecture

From the beginning it should be noticed that the system operates inside a surrounding manufacturing environment, including the physical space, the cyber space, the social environment, and the commercial environment [16]. All the assets needed in order to carry out the manufacturing process are coming from these spaces and environments: informatic modules and knowledge (selected from the cyber offer), physical modules and energy (selected from the physical offer), and personnel (selected from the existing human resources). As consequence of a certain product demand, the system receives an order involving the task $T_{n}$, which is stored in scheduled tasks pool $T$. At the moment when the system passes to $T_{n}$ accomplishment, both making-holon and learning-holon successively execute their functions, as presented in previous section. Thus, the deliverable $D_{\alpha}$ is obtained, while both new knowledge and knowhow generated as result of the performed manufacturing process are returned to manufacturing environment.

\subsection{System holarchies}

Because, in general, a single holon (no matter if making- or learning-one) cannot accomplish the entire task, this has to collaborate with other holons, by task configuring / reconfiguring. This way, successive generations of holons take birth and an architectural holarchy of the system is generated (Figure 4). Each holon belonging to this holarchy has assigned its specific support, which, associated with the received task, defines the holon [16].

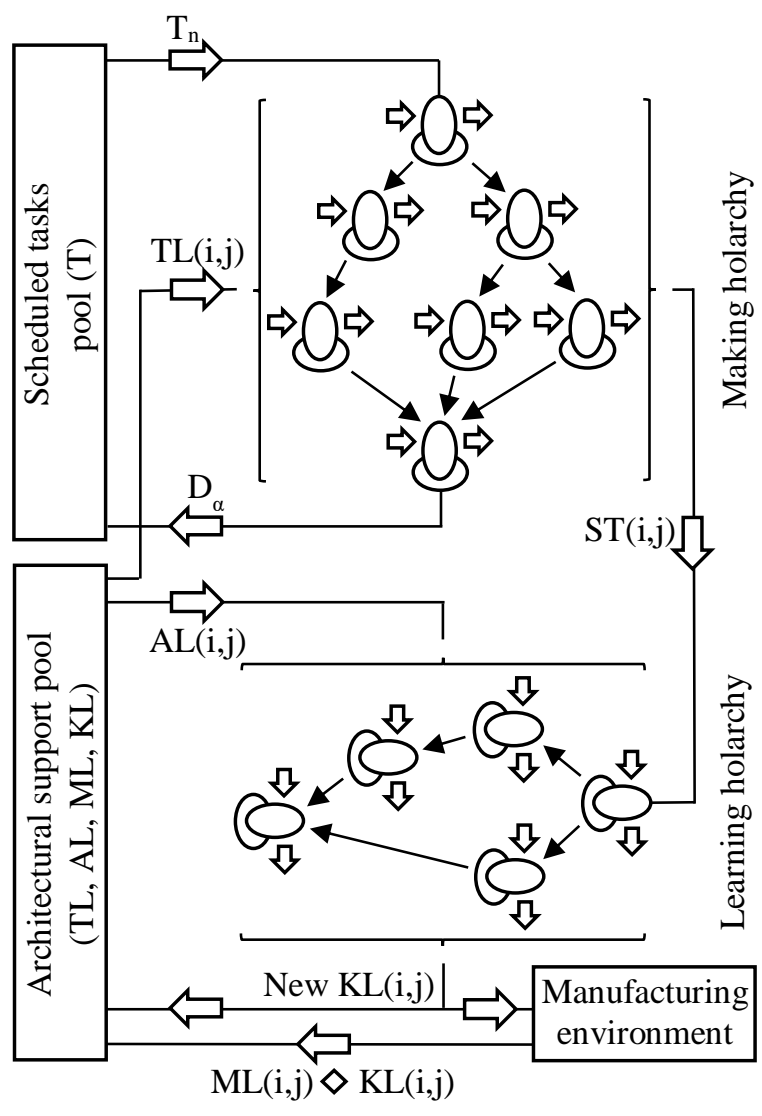

Fig. 4. System holarchies

The architectural holarchy of the system is actually composed by two distinct holarchies: the makingholarchy (among the making-holons) and the learning-holarchy (among the learning-holons). The 
connection between these holarchies is the statement $S T(i, j)$ that the first one submits to the second one as result of $T_{n}$ accomplishment. Each of the two holarchies has the same type of inputs/outputs as the corresponding type of holon (see Figure 4).

\section{HOLISTIC MONITORING}

In manufacturing, the conventional monitoring target is to find the state of the monitored system/process by continuously looking after the values of a given set of variables, previously established.

The holistic monitoring, which should be understood as an enhanced version of monitoring, aims a double target: the state monitoring (seen as a more comprehensive form of the conventional monitoring), and the dynamics monitoring (seen as higher level of the monitoring action, during which new knowledge is extracted on the base of state monitoring results).

\subsection{State monitoring}

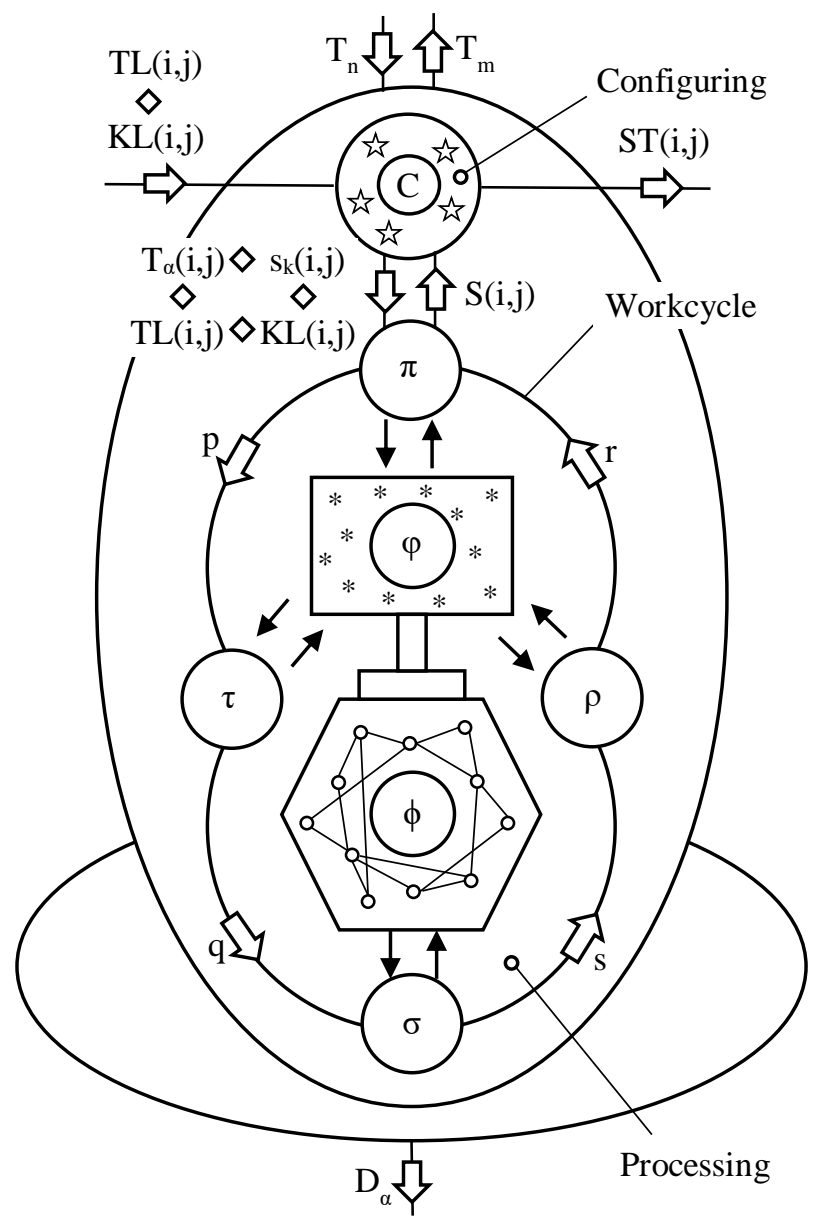

Legend: o - system state variable; - - causal relation; * - value of state variable; $\_$- system state.

Fig. 5. State monitoring

The system state monitoring is in connection to the operation of the involved making-holons. For simplicity, the case of a manufacturing system comprising a unique making-holon (Figure 5) will be further addressed.

The making-holon accomplishes its manufacturing function as described in section 2.2.

In Configuring stage, after establishing the assumed task $T_{\alpha}$ and assigning to it the class and the tool version, $T_{\alpha} \rightarrow T_{\alpha}(i, j)$, the holon also defines the set of variables $\left\{s_{k}(i, j)\right\}$ by which the state of the system follows to be described.

Six modules are involved in Processing stage. They are cyclically operating, workcycle after workcycle, information being exchanged among them, as vectors comprising values of system state variables.

At the beginning of each workcycle, the piloting module $\pi$ establishes the current values for a set of variables selected in order to define the processing workcycle target. These values are found after analysing the results of the previous workcycle and compose the piloting data-vector $p$, which is transmitted to programming module $\tau$. This module decides about the values of the variables that are required for setting the manufacturing modules operation according to the currently followed target. The driving module $\sigma$ receives the setting data-vector $q$ and, on this base, it controls the functioning of doing module $\phi$. The doing module operation is monitored by sensors, which generate the sensing data-vector $s$ (composed by values of system state variables) and submit it to measuring module $\rho$. The measuring module evaluates the results of current workcycle and generates the result data-vector $r$, which is sent to piloting module. At the same time, it associates the values of result-variables to the corresponding values of system state variables and sends this information to monitoring module $\varphi$, to be stored for future use.

The reunion of $p, q, r$ and $s$ vectors, recorded for a given workcycle, defines the system current state $S(i, j)$ corresponding to that workcycle.

\subsection{Dynamics monitoring}

The system dynamics monitoring is in connection to the operation of the involved learning-holons. The case of a unique learning-holon (Figure 6) will be further addressed. The learning-holon accomplishes its manufacturing function as described in section 2.2. In Aggregating stage, the holon builds a dataset $D S(i, j)$ by processing through specific procedures [17] the information received as $S T(i, j)$ statements.

The same types of modules as the ones involved in Processing stage are cooperating in order to perform the Modeling stage workcycle. However, their jobs are different, according to the specificities of modeling relative to processing. Thus, the target established by piloting refers to modeling subject, the effect-variables selected to describe it, the model 
performance that is required, the acceptable causevariables to be used in model construction, and no longer to the output of the manufacturing process. "Programming" deals with specifying the settings of the modeling algorithm. The driving and the doing modules (last one denoted, this time, by $\Omega$ ) are of numeric (instead of manufacturing) type, while the result of modeling algorithm application is the finding of pursued causal models. In Modeling stage, "measuring" means to find the values of selected effect-variables by applying the previously found causal models. The set of values for all the state variables as resulted from current modeling workcycle are transmitted to monitoring module $\omega$, while the result data-vector comprises the values describing all the components of the system model $M(i, j)$.

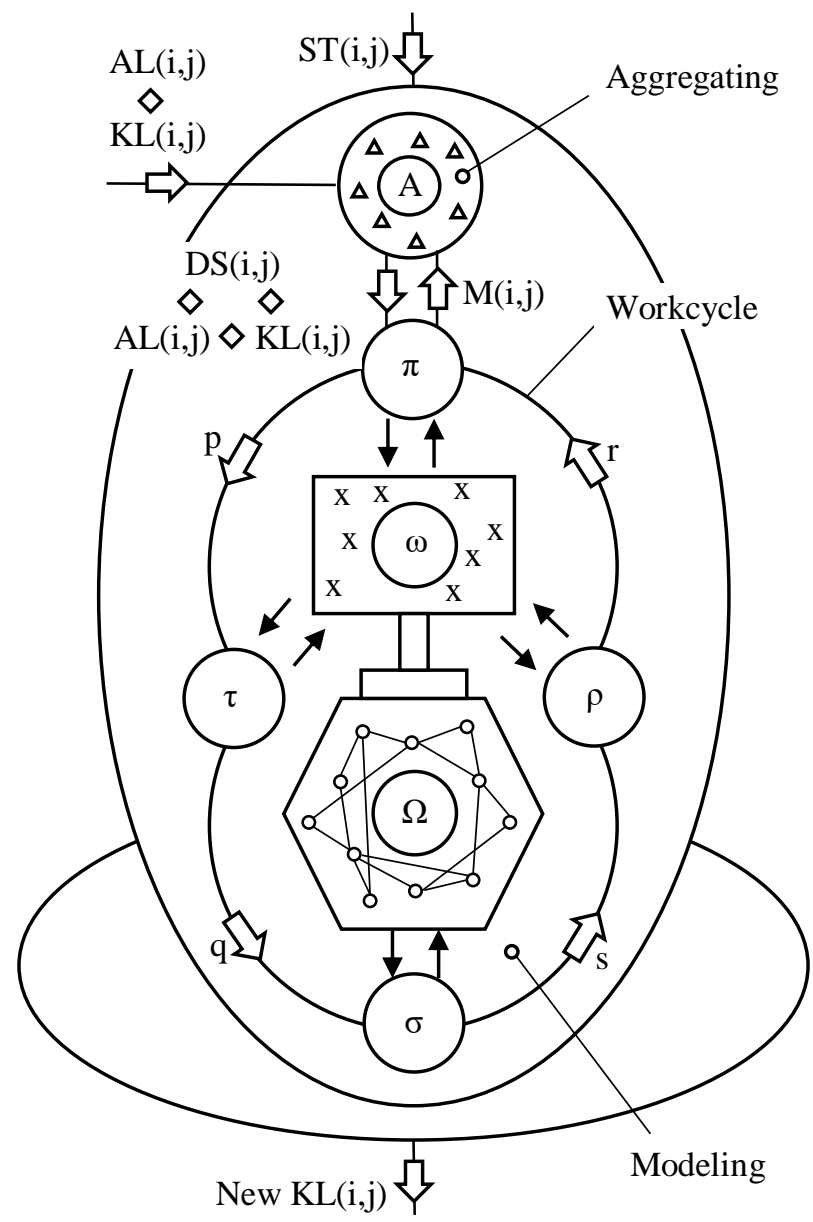

Legend: o - system state variable; - - causal relation; $\mathrm{X}$ - set of state variables values; $\boldsymbol{\Delta}$ - system model.

Fig. 6. Dynamics monitoring

\subsection{Illustrative example}

An illustrative example addressing the case of a turning task $T_{\alpha}$ is further presented in order to facilitate the understanding of the holistic monitoring concept $\&$ application process.

The graphical description of $T_{\alpha}$ is depicted in Figure 7. In configuring stage, $T_{\alpha}$ is identified as being of $i=$

3 class and partitioned in two sub-tasks $T_{A}$ and $T_{B}$, which are going to be accomplished with the same version of tooling, $j=8$. Hereby, $T_{\alpha}=T_{a}(3,8)$.

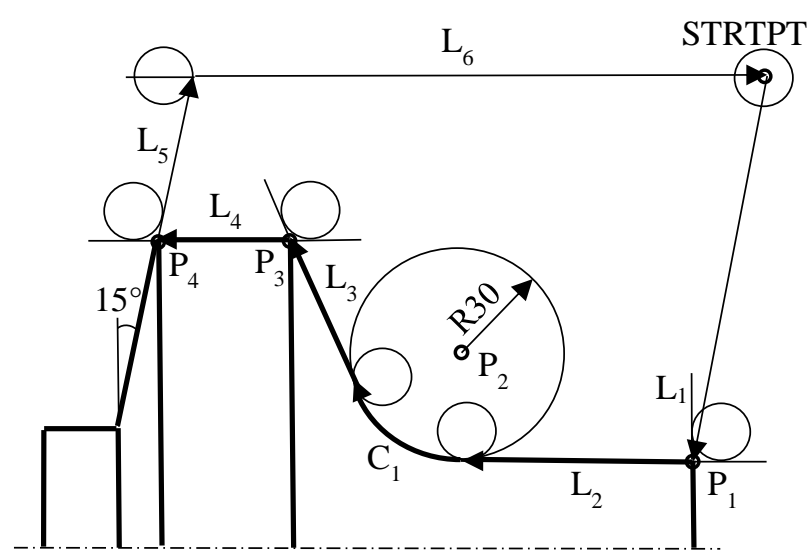

Task $\mathrm{T}_{\mathrm{A}}=\left(\right.$ STRTPT, P3), $\left\{\sigma, \mathrm{a}, \mathrm{R}_{\mathrm{a}}, \delta, \mathrm{C}, \mathrm{T}, \mathrm{E}\right\}\left(\mathrm{T}_{\mathrm{A}}\right)$

Task $\mathrm{T}_{\mathrm{B}}=(\mathrm{P} 3, \mathrm{STRTPT}),\left\{\sigma, \mathrm{a}, \mathrm{R}_{\mathrm{a}}, \delta, \mathrm{C}, \mathrm{T}, \mathrm{E}\right\}\left(\mathrm{T}_{\mathrm{B}}\right)$

Fig. 7. Illustrative example for task configuring - graphical description of the task

The numerical description of the same task is presented below, as list of instructions written in a modified version of APT programming language, adapted to the requirements of the new generation of manufacturing systems.

\section{\$ Conditions}

$\mathrm{CND} 1=\mathrm{COND} / \sigma, \mathrm{a}, \mathrm{R}_{\mathrm{a}}, \delta, \mathrm{C}, \mathrm{T}, \mathrm{E}$

$\mathrm{CND} 2=\mathrm{COND} / \sigma, \mathrm{a}, \mathrm{R}_{\mathrm{a}}, \delta, \mathrm{C}, \mathrm{T}, \mathrm{E}$

\$\$ Geometry

STRPT $=$ POINT $/ 160,100$

$\mathrm{P} 1=\mathrm{POINT} / 120,200$

$\mathrm{P} 2=\mathrm{POINT} / 100,50$

$\mathrm{P} 3=\mathrm{POINT} / 40,70$

$\mathrm{P} 4=\mathrm{POINT} / 10,70$

C1 = CIRCLE / CENTER, P2, RADIUS, 30

L1 = LINE / P1, PARLEL, YAXIS

L2 = LINE / P1, LEFT, TANTO, C1

L3 = LINE / P3, RIGHT, TANTO, C1

L4 = LINE / P4, PERLEL, XAXIS

L5 = LINE / P4, ATANGL, 15, YAXIS

L6 = LINE / STRPT, PARLEL, XAXIS

\$ Kinematics

SET / CND1

RAPID

FROM / STRPT

GO/TO, L1, TO, L3

TLRGT, GORGT / L2, PAST, C1

GOFWD / C1, TANTO, L3

GOFWD / L3, PAST, L4

SET / CND2

GOLFT / L4, PAST, L5

RAPID

GORGT / L5, ON, L6

GO/ON / STRPT

FINI 
Then, according to state monitoring procedure, the set of state variables $s_{k}$ is defined. It is composed by the variables corresponding to the tasks of $i=3$ class, which, in this case, are supposed to be:

$$
\left\{t_{k}(3)\right\}=\left\{\sigma, a, R_{a}, \delta, C, T, E\right\}
$$

and the variables of the $j=8$ version of tooling used for accomplishing this class of tasks, supposed to be:

$$
\begin{gathered}
\left\{t l_{k}(3,8)\right\}=\{C T, s, f\}, \\
\left\{s_{k}(3,8)\right\}=\left\{t_{k}(3)\right\} \cup\left\{t l_{k}(3,8)\right\} .
\end{gathered}
$$

In relations (5) and (6), $\sigma$ means the cutting strength of the machined material in MPa, $a$ - the thickness of the detached layer in $\mathrm{mm}, R_{a}$ and $\delta$-the roughness and the deviation of the generated surface in $\mu \mathrm{m}$ and mm, respectively, $C, T$ and $E-$ the specific cost, machining time and consumed energy in Euro/ $\mathrm{cm}^{3}$, $\mathrm{min} / \mathrm{cm}^{3}$ and $\mathrm{kWh} / \mathrm{dm}^{3}$, respectively, $C T$ - the cutting tool indicative, $s$ - the cutting speed in $\mathrm{m} / \mathrm{min}$, and $f-$ the feed in $\mathrm{mm} / \mathrm{rev}$.

In what concerns the structure of the data-vectors which circulate between the modules of the makingholon, let us select $C, T, \sigma$ and $a$ (for piloting vector $p$ ), $C T, s$ and $f$ (for setting vector $q$ ), $F$ and $\delta$ (for

\begin{tabular}{|c|c|c|c|c|c|c|c|c|c|c|c|c|c|c|c|c|c|}
\hline \multicolumn{2}{|c|}{ Task } & Support & \multicolumn{15}{|c|}{ New knowledge } \\
\hline Symbo & Class & $(\mathrm{i}, \mathrm{j})$ & \multicolumn{15}{|c|}{$(i, j)$} \\
\hline \multirow{12}{*}{$\mathrm{T}_{\alpha / \mathrm{n}}$} & \multirow{12}{*}{ (3) } & \multirow{12}{*}{$(3,8)$} & \multirow{12}{*}{$\begin{array}{c}\text { New } \\
\operatorname{KL}(3,8)\end{array}$} & \multirow{2}{*}{\multicolumn{2}{|c|}{$\mathrm{S}(3,8)$}} & \multicolumn{4}{|c|}{$\mathrm{p}$} & \multicolumn{3}{|c|}{$\mathrm{q}$} & \multicolumn{2}{|c|}{$\mathrm{s}$} & \multicolumn{3}{|c|}{$\mathrm{r}$} \\
\hline & & & & & & $\mathrm{C}$ & $\mathrm{T}$ & $\sigma$ & $\mathrm{a}$ & CT & $\mathrm{s}$ & $\mathrm{f}$ & $\mathrm{F}$ & $\delta$ & $\mathrm{C}$ & $\mathrm{T}$ & $\mathrm{E}$ \\
\hline & & & & \multirow{6}{*}{\multicolumn{2}{|c|}{$\operatorname{DS}(3,8)$}} & 0.03 & 0.055 & 370 & 3 & $\mathrm{~T} 2$ & 78 & 0.22 & 171 & 0.012 & 0.033 & $\mid 0.059$ & 1.08 \\
\hline & & & & & & 0.04 & 0.07 & 370 & 3.4 & $\mathrm{~T} 7$ & 90 & 0.14 & 136 & 0.009 & 0.038 & 0.069 & 1.22 \\
\hline & & & & & & 0.02 & 0.025 & 500 & 4.5 & $\mathrm{~T} 3$ & 130 & 0.2 & 220 & 0.014 & 0.014 & 0.025 & 0.7 \\
\hline & & & & & & 0.035 & 0.06 & 480 & 3.1 & $\mathrm{~T} 7$ & 98 & 0.15 & 123 & 0.01 & 0.037 & 0.068 & 1.18 \\
\hline & & & & & & 0.11 & 0.2 & 400 & 1.05 & $\mathrm{~T} 2$ & 70 & 0.21 & 57.6 & 0.007 & 0.107 & 0.194 & 1.9 \\
\hline & & & & & & 0.035 & 0.06 & 420 & 4.2 & $\mathrm{~T} 1$ & 140 & 0.09 & 109 & 0.011 & 0.032 & 0.058 & 1.14 \\
\hline & & & & \multirow{4}{*}{$\mathrm{M}(3,8$} & $\overline{\mathrm{X}}$ & 0.045 & 0.078 & 423.3 & 3.21 & - & 101 & 0.17 & 136 & 0.0105 & 0.043 & 0.079 & 1.20 \\
\hline & & & & & SD & 0.029 & 0.056 & 50.5 & 1.11 & - & 25.7 & 0.045 & 50.5 & 0.002 & 0.029 & 0.053 & 0.35 \\
\hline & & & & & $\mathrm{a}$ & - & - & -1 & \begin{tabular}{|l|}
-0.03 \\
\end{tabular} & - & $5-\mathrm{E}-4$ & 0.048 & $-E-4$ & - & $*$ & - & - \\
\hline & & & & & $\mathrm{b}$ & - & - & -E-4 & \begin{tabular}{|l|}
-0.07 \\
\end{tabular} & - & 0.001 & 0.011 & - & - & - & $*$ & - \\
\hline
\end{tabular}
sensing vector $s$ ), $C, T$ and $E$ (for result vector $r$ ).

Table 1. Illustrative example

The holistic monitoring result is new knowledge which, in the addressed turning task, can be synthesized in the form presented in Table 1. Each line giving a set of $p, q, s$ and $r$ values describes the system state for one task accomplishment. On the base of the dataset resulted after recording a certain number of such lines, the learning-holon processes the included information and provides the system model. Here, the model comprises three issues:

- $\bar{X}$, meaning a vector that includes the average value of each state variable,

- $S D$ - a vector that includes the standard deviation for each state variable, and

- $a, b-$ sets of coefficients defining the numerical models of the result-variables, namely $C$ and $T$.

The proposed models are linear and they can be obtained by multiple regression. The variables used to describe the result-variable must be selected by causal identification [17]. For example, in the specific cost case, the model shape is:

$$
C=c_{1} \cdot a+c_{2} \cdot s+c_{3} \cdot f+c_{4} \cdot F+c_{5},
$$

It should be noticed that the identified coefficients mean a qualitative measure of the causal relations.

\section{CONCLUSIONS}

Starting from the challenges arising in front of the next generation of machining systems, the conceptual rebuilding of the monitoring system is needed. The here-introduced holistic monitoring concept lays on a holonic modeling of the manufacturing system based on the existence of two types of holons (making- and learning-holons) and on a specific holarchy.

The holistic monitoring means evaluation and revealing of machining system current state \& dynamics. In other words, the holistic monitoring concerns both the values of the variables describing system state and the causality relations among them.

The entire information resulted after each machining task accomplishment should be delivered as knowledge, which could further enable to operate the machining system according to a new paradigm: the knowledge-based machining.

\section{REFERENCES}

1. Gostev, I., Golosov, P. (2020). About one quality monitoring of a surface of gear wheels, International Journal of Modern Manufacturing Technologies, XII(3), pp. 41-46. 
2. Xing, K., Liu, X., Liu, Z., Mayer, J.R.R, Achiche, S. (2021). Low-Cost Precision Monitoring System of Machine Tools for SMEs, Proc. CIRP 96, pp. 347352.

3. Boca M., Horodincă M. (2020). The possibilities to use a signal for describing the elastic deflection behaviour of a workpiece processed by turning, International Journal of Modern Manufacturing Technologies, XII(2), pp. 17-22.

4. Lee, D.E., Hwang, I., Valente, C.M.O., Oliveira, J.F.G., Dornfeld, D.A. (2006). Precision manufacturing process monitoring with acoustic emission, Int Journal of Machine Tool \& Manufacture 46, pp. 176-188.

5. Behrens, B.-A., Hubner, S., Wolki, K. (2017). Acoustic emission - A promising and challenging technique for process monitoring in sheet metal forming, J. Manuf. Process 29, pp. 281-288.

6. Pandiyan, V., Shevcsik, S., Wasmer, K., Castagne, S., Tjahjowidodo, T. (2020). Modelling and monitoring of abrasive finishing processes using artificial intelligence techniques: A review, J. Manuf. Process 57, pp. 114-135.

7. Garcia Plaza, E., Nunez Lopez, P.J., Beamud Gonzales, E.M. (2019). Efficiency of vibration signal feature extraction for surface finish monitoring in CNC machining, J. Manuf. Process 44, pp. 145-157. 8. Lauro, C.H., Brandao, L.C., Baldo, D., Reis, R.A., Davim, J.P. (2014). Monitoring and processing signal applied in machining processes $-A$ review, Measurement 58, pp. 73-86.

9. Sevilla-Camacho, P.Y., Robles-Ocampo, J.B., Jauregui-Correa, J.C., Jimenez-Villalobos, D. (2015). FPGA-based reconfigurable system for tool condition monitoring in high-speed machining process, Measurement 64, pp. 81-88.

10. Bhuiyan, M.S.H., Choudhury, I.A., Dahari, M. (2014). Monitoring the tool wear, surface roughness and chip formation occurrences using multiple sensors in turning, J. Manuf. Syst. 33, pp. 476-487.

11. Zhao, R., Yan, R., Chen, Z., Mao, K., Wang, P., Gao, R.X. (2019). Deep learning and its applications to machine health monitoring, Mechanical Systems and signal processing 115, pp. 213-237.

12. Xing, K., Achiche, S., Mayer, J.R.R. (2019). Five-axis machine tools accuracy condition monitoring based on volumetric errors and vector similarity measures, International Journal of Machine Tool \& Manufacture 138, pp. 80-93.

13. Assad, F., Konstantinov, S., Nureldin, H., Waseem, M., Rushforth, E., Ahmad, B., Harrison, R. (2021). Maintenance and digital health control in smart manufacturing based on condition monitoring, Procedia CIRP 97, pp. 142-147.

14. Lv, L., Deng, Z., Liu, T., Li, Z., Liu, W. (2020). Intelligent technology in grinding process driven by data: A review, Journal of Manufacturing Processes 58, pp. 1039-1051.

15. Liao, Y., Ragai, I., Huang, Z., Kerner, S. (2021). Manufacturing process monitoring using timefrequency representation and transfer learning of deep neural networks, Journal of Manufacturing Processes 68, 231-248.

16. Frumuşanu, G., Epureanu, A. (2021). Architectural Holarchy of the Next Generation Manufacturing System, accepted for publication in International Journal of Modeling and Optimization. 17. Frumuşanu, G., Afteni, C., Epureanu, A. (2021). Data-Driven Causal Modeling of the Manufacturing System, Transactions of FAMENA 45(1), pp. 43-62.

Received: March 09, 2021 / Accepted: December 20, 2021 / Paper available online: December 25, 2021 (C) International Journal of Modern Manufacturing Technologies 